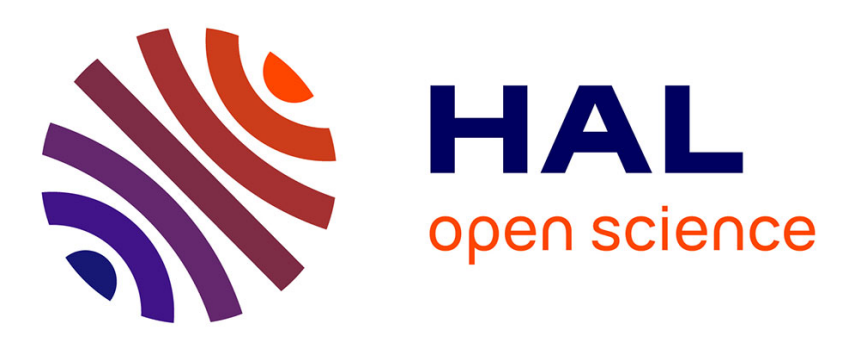

\title{
Structural features of pyrocarbon atomistic models constructed from transmission electron microscopy images
}

Jean-Marc Leyssale, Jean-Pierre da Costa, Christian Germain, Patrick Weisbecker, Gérard Vignoles

\section{To cite this version:}

Jean-Marc Leyssale, Jean-Pierre da Costa, Christian Germain, Patrick Weisbecker, Gérard Vignoles. Structural features of pyrocarbon atomistic models constructed from transmission electron microscopy images. Carbon, 2012, 50 (12), pp.4388-4400. 10.1016/j.carbon.2012.05.015 . hal-00773213

\section{HAL Id: hal-00773213 https://hal.science/hal-00773213}

Submitted on 11 Jan 2013

HAL is a multi-disciplinary open access archive for the deposit and dissemination of scientific research documents, whether they are published or not. The documents may come from teaching and research institutions in France or abroad, or from public or private research centers.
L'archive ouverte pluridisciplinaire HAL, est destinée au dépôt et à la diffusion de documents scientifiques de niveau recherche, publiés ou non, émanant des établissements d'enseignement et de recherche français ou étrangers, des laboratoires publics ou privés. 


\title{
Structural features of pyrocarbon atomistic models constructed from transmission electron microscopy images
}

\author{
J.-M. Leyssale ${ }^{\mathrm{a}, *}$, J.-P. Da Costa ${ }^{\mathrm{b}}$, C. Germain ${ }^{\mathrm{b}}$, P. Weisbecker ${ }^{\mathrm{a}}$, G. L. \\ Vignoles $^{\mathrm{c}}$ \\ ${ }^{a}$ CNRS, Laboratoire des Composites ThermoStructuraux, UMR 5801 CNRS-Snecma \\ Propulsion Solide-CEA-Université Bordeaux 1,33600 Pessac, France \\ ${ }^{b}$ Univ. Bordeaux, ENITAB, Laboratoire de l'Intégration du Matériau au Système, UMR \\ 5218 CNRS-IPB - Université Bordeaux 1, 33405 Talence, France \\ ${ }^{c}$ Univ. Bordeaux, Laboratoire des Composites ThermoStructuraux, UMR 5801 \\ CNRS-Snecma Propulsion Solide-CEA-Université Bordeaux 1, 33600 Pessac, France
}

\begin{abstract}
We report on atomistic models of laminar pyrocarbons constructed using a combination of 2D high resolution transmission electron microscopy (HRTEM) lattice fringe image analysis, 3D image synthesis and atomistic simulated annealing. In a first step, the effectiveness of the method and the convergence of the models with respect to the quench rate are checked on small systems. Then, the nanostructural features of large fully carbonaceous atomistic models obtained from the HRTEM images of a rough laminar pyrocarbon, asprepared and after partial graphitization, are discussed. Both models show a very pronounced $s p^{2}$ character $(\geq 97 \%)$, essentially made of hexagonal rings $(\geq 88 \%)$ and pentagonal and heptagonal rings in similar amounts $(\approx 6 \%)$. The latter mostly form pentagon-heptagon pairs or networks of line defects
\end{abstract}

\footnotetext{
*Corresponding author

Email address: leyssale@lcts.u-bordeaux1.fr (J.-M. Leyssale)
} 
between misoriented hexagonal domains. Numerous pairs of screw dislocations, connecting different graphene domains, are also observed while edge dislocations with unsaturated carbon atoms are almost absent. The models are validated with respect to experimental pair distribution functions, showing excellent agreement.

\section{Introduction}

Low temperature pyrolytic carbons, or pyrocarbons (PyCs), as obtained from chemical vapor infiltration of fibrous preforms, are essential constituents of many high performance composite materials; such as the matrices of Carbon/Carbon composites [1] for spatial applications or as the interphases of many $\mathrm{SiC} / \mathrm{SiC}$ materials found in aeronautic or nuclear energy industries [2]. These pyrocarbons are dense $s p^{2}$ carbons but, in their "as prepared" form, can contain large and variable amounts of defects. Different classes of PyCs, differing in their microstructure and consequently in their properties (graphitizability, mechanical properties, thermal properties, etc...), have been identified [3-5] and related to the preparation conditions [6, 7]. Understanding their structure-property relationship is a key issue in quality control as well as in designing new materials with targeted properties. Many papers have been published on the different microstructures of PyCs and their properties [3-9]. However, very few of them have given a clear picture of these materials at the atomic scale (nanostructure) so far, and, for instance, the first pair distribution functions (PDFs) of PyCs have only been measured very recently [10].

Atomistic modeling is a particularly attractive approach to determine, 
and also visualize, the nanostructure of such partially disordered materials and two classes of methods, the mimetic and knowledge based approaches [11], can be used in that goal.

In the mimetic approach, atomistic models are built by simulating the synthesis process of the materials. For instance, liquid quench molecular dynamics (LQMD) simulations are often used to mimic the physical vapor deposition (PVD) of amorphous carbons $[12,13]$. However, direct MD simulation is limited to extremely fast synthesis processes, as this technique cannot span timescales larger than a microsecond. Applying a mimetic method to a "slow" fabrication process thus requires developping problem-specific approaches $[14,15]$.

In the knowledge based approach, on the other hand, no attention is paid to the history of the materials; the models are solely built on the basis of reproducing a selected set of properties. The most popular technique in that purpose is the reverse Monte Carlo (RMC) method [16]. In a RMC simulation, atoms, or blocks of rigidly bound atoms, are randomly moved in space and the moves are either accepted (or rejected) with a probability based on the root mean square deviation between the PDF of the model and the experimental PDF of the material. Among the recent improvements of the RMC method one can site the hybrid RMC (HRMC) method, a composite method between RMC and conventional Metropolis Monte Carlo (MC) in which an empirical reactive potential ensures the formation of properly defined chemical bonds in addition to the fit of the PDF $[17,18]$. Also, PDF data can be complemented, or even replaced, by other sets of structural information in an RMC (or HRMC) process. For instance, Nguyen et al. have 
constructed models of activated carbons using a HRMC method based on the reproduction of pore size and pore wall thickness distributions [19].

In the particular case of highly textured materials, a successful knowledge based construction must be based, at least in part, on the reproduction of some nanotextural information. Lattice fringe images obtained in high resolution transmission electron microscopy experiments are often used to describe the nanotexture of dense aromatic carbons [3]. Considering this, we immediately think of an RMC (or HRMC) process in which the target experimental data would be the HRTEM image. Even though it is possible to directly simulate a HRTEM image from an atomistic configuration - it has even been done for validation purposes in some papers [20, 21] - image simulation is much too computationally expensive to be performed, say, tens of thousands times, as would be required in an RMC simulation.

Following recent progress in statistical analysis of HRTEM images [22-26] and in image synthesis techniques $[27,28]$ we have recently proposed a new knowledge based method, the image guided atomistic reconstruction (IGAR) method [29], aiming at building realistic atomistic models of nanotextured carbons from almost the sole knowledge of their HRTEM images and density.

This method is presented in details in section 2. Results of its application to two images of a rough laminar $\mathrm{PyC}$ are then presented in section 3 with a particular emphasis on both the structural features of the obtained models and on the parameters - inherent to the methodology - that can affect the quality of the resulting models. Finally we conclude in section 4 with a discussion on the "hits and misses" of the proposed approach and of some possible ideas of improvement. 


\section{Methods}

\subsection{HRTEM-like 3D image synthesis}

The first step of the IGAR method consist in building 3D analogues of the actual 2D HRTEM specimens. To do so we extend to 3D the parametric approach of Portilla and Simoncelli [27] for the synthesis of a 2D texture from a 2D exemplar. First, after being filtered with both a radial and directional band-bass filter [30], them HRTEM image sample is decomposed into a pyramid of multi-resolution subbands which are then analyzed to produce a pyramidal collection of 2D first order (mean, variance, skewness and kurtosis) and second order (autocorrelation coefficients) reference statistics [27]. In a second step, these 2D reference statistics are extended to produce a pyramical collection of $3 \mathrm{D}$ target statistics through $2 \mathrm{D} / 3 \mathrm{D}$ statistical inference. This is trivial for first order statistics which are equal in 2D and $3 \mathrm{D}$.

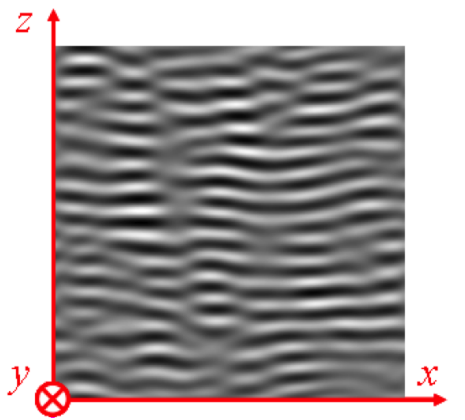

a)

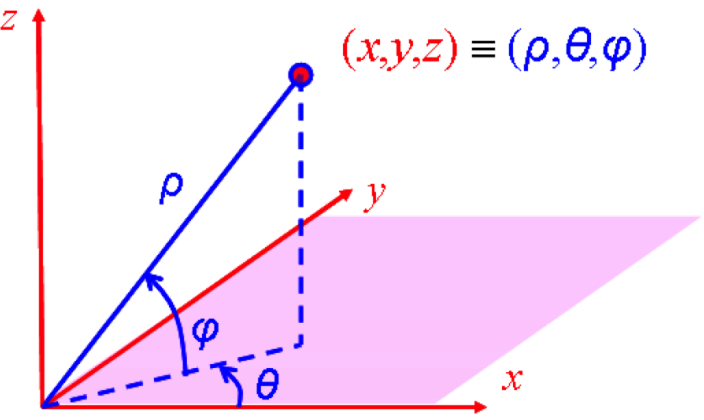

b)

Figure 1: Filtered HRTEM image sample (a) and the chosen Cartesian $(x, y, z)$ and spherical $(\rho, \theta, \phi)$ coordinate systems (b). 
Fig. 1 helps us in explaining how we deduce 3D autocorrelation coefficients $r_{3 D}(x, y, z)$ from the reference $2 \mathrm{D}$ autocorrelation coefficients $r_{2 D}(x, z)$. Laminar pyrocarbons are orthotropic materials: all the properties of the material (structural, mechanical, thermal, etc...) are the same whatever the direction considered provided that it is orthogonal to the orthotropy direction, the stacking direction of graphene layers (vertical direction in Fig. 1a). This, of course, also holds for the properties of HRTEM images, and so $r_{3 D}^{s p h}(\rho, \theta, \varphi)=r_{2 D}^{p o l}(\rho, \varphi)$, whatever the value of $\theta$ (here superscripts $s p h$ and pol relate to respectively spherical and polar coordinate systems (see Fig. 1b)). It easily follows [28] that the $3 \mathrm{D}$ autocorrelations coefficients $r_{3 D}$ can be deduced from $r_{2 D}$ by interpolation using

$$
r_{3 D}(x, y, z)=r_{2 D}(\rho \cos \varphi, \rho \sin \varphi)
$$

Finally, a 3D texture, initially random, is alternately decomposed into a 3D multi-resolution pyramid, modified to meet the 3D target statistics, reconstructed, re-decomposed, etc... until convergence. At that point we dispose of a 3D analogue of the filtered experimental HRTEM image in the sense that every $2 \mathrm{D}$ slice of the $3 \mathrm{D}$ image, taken parallel to the orthotropy axis, is statistically equivalent to the latter.

\subsection{Image guided atomistic reconstruction}

The IGAR procedure starts with the definition, or the superposition, of a physical system on the synthesized 3D images. Basically, a physical volume, here a cube, with periodic boundary conditions (3D images are periodic as well) and edge size given by the interfringe distance of the material $\left(d_{002}\right)$ multiplied by the average number of fringes in the stacking direction, is 
associated to the image blocks. This volume is then filled with randomly located carbon atoms at the suited density. Then, we perform a simulated annealing (a "slow" temperature quench) simulation, using either molecular dynamics (MD) or Monte Carlo (MC) sampling, in which the potential energy of the system is defined by

$$
\mathcal{U}=\mathcal{U}_{R E B O}+\mathcal{U}_{H R T E M}
$$

In this equation $\mathcal{U}_{R E B O}$ represents the interatomic potential energy, defined by the second generation REBO potential function [31]:

$$
\mathcal{U}_{R E B O}=\sum_{i=1}^{N-1} \sum_{j=i+1}^{N} f_{c}\left(r_{i j}\right)\left[V_{R}\left(r_{i j}\right)-b_{i j} V_{A}\left(r_{i j}\right)\right]
$$

where $N$ is the number of atoms, $r_{i j}$ is the distance between carbon atoms $i$ and $j, f_{c}(r)$ is a switching function that goes smoothly from unity for $r=0.17 \mathrm{~nm}$ to zero for $r=0.2 \mathrm{~nm}, V_{R}(r)$ and $V_{A}(r)$ are respectively the repulsive and attractive potentials and $b_{i j}$ is an empirical bond order term taking into account the local environments of atoms $i$ and $j$. $\mathcal{U}_{\text {HRTEM }}$ is a fictitious interaction potential between the physical system and the 3D HRTEM image:

$$
\mathcal{U}_{\text {HRTEM }}=k_{\text {Im }} \sum_{i=1}^{N} I_{\text {Im }}\left(\mathbf{r}_{\mathbf{i}}\right)
$$

where $I_{I m}\left(\mathbf{r}_{\mathbf{i}}\right)$ is the greyscale level, ranging from zero to one for respectively black and white voxels, of the $3 \mathrm{D}$ image at position $\left(\mathbf{r}_{\mathbf{i}}\right)$ of atom $i\left(I_{I m}\left(\mathbf{r}_{\mathbf{i}}\right)\right.$ is computed by trilinear interpolation using the greyscale levels of the eight voxels closest to location $\mathbf{r}_{\mathbf{i}}$ ). 
This last term is rather unusual and definitely unphysical. Its only aim is to bring the atoms to settle on the dark areas of the 3D images (the fringes). It actually acts like an external potential field imposing an experiment based property of the materials to the model, namely its nanotexture, in close similarity with the HRMC method in which the RMC part (the fit to the PDF) can be somehow considered as an external potential as well. IGAR somehow resembles the one work of Petersen et al. [32] who performed HRMC simulations in which atoms were constrained on analytic surfaces derived from HRTEM observations to build atomistic models of glassy carbons. However, in the IGAR method atoms are not rigidly constrained on the fringes, although being attracted by the dark areas, and no PDF data are used in the model construction process, unlike what is done in most RMC methods. Also, we can note a certain similarity between the IGAR approach and the templating approach of Roussel et. al. [15]. Indeed, while they simulate the equilibrium between an ideal carbon gas and the carbon phase sorbed in the zeolite, or in other words the carbon gas in presence of the "external" potential field created by the zeolite, we simulate the quench of a carbon liquid in presence of an external potential field created by the 3D HRTEM image.

There are three "user-defined" parameters in the IGAR procedure: (i) the initial temperature of the system $T_{0}$; (ii) the rate (in $\mathrm{K} / \mathrm{s}$ or $\mathrm{K} / \mathrm{MC}$ attempt for simulated annealing performed with respectively MD or MC simulations) at which the system is cooled down to zero temperature; and (iii) the proportionality factor $k_{I m}$ between grey level and energy. In order to avoid any bias from the initial configuration of the system, $T_{0}$ has to 
be chosen so that the system at this temperature is diffusing (liquid). The quench rate has to be chosen as slow as possible to ensure convergence of the final configurations, in terms of energies and structure, while remaining computationally affordable. Finally, $k_{I m}$ has to be strong enough to impose the suited nanotexture while being as low as possible in order not to affect the chemical quality of the models (here we remember that $\mathcal{U}_{R E B O}$ is physical, $\mathcal{U}_{\text {HRTEM not). }}$

\subsection{Simulation details}

Two experimental HRTEM images taken from the matrices of $\mathrm{C} / \mathrm{C}$ composites were considered (see Fig. 2a and b). They correspond respectively to a rough laminar PyC, as prepared (AP) and after heat treatment (HT) inducing a partial graphitization. These images, on which the 2D analysis is performed have been choosen from the domains with highest contrast among larger samples, to insure the best possible alignment with regards to the Scherzer focus and Eucentric position, although our aim here is mainly to discuss the similarities and differences between such an AP and HT materials and not to quantitatively characterize the two individual materials. From these 2D images, four 3D cubic image blocks, $128 \times 128 \times 128$ voxels each were obtained through the 2D analysis - 3D synthesis image processing scheme described in section 2.1. The first two (one for each material) are small images, 13 fringes high on average along the orthotropy axis, aimed at finding the most suitable cooling sequence and optimum value of $k_{I m}$. The other two, counting on average respectively 16 and 17 fringes for respectively the AP and HT PyCs (see Fig. 2e and f), are then used in order to build models of sufficient size to capture the nanostructural differences between 
the two materials. The steerable pyramid decomposition, used in the image analysis synthesis step, was performed with 3 levels and 4 orientations and autocorrelation coefficients were taken on $7 \times 7$ neighborhoods. Ten iterations were enough to converge towards stable 3D textures. Then, the resolution of the synthesized textures was improved to $512 \times 512 \times 512$ through spline interpolation before feeding the IGAR code.

In the IGAR procedure, we consider the materials as entirely made of carbon atoms (ideally around one atomic percent of hydrogen should be considered as well [10]) with an equal density of $2.1 \mathrm{~g} / \mathrm{cm}^{3}$ (a higher (resp. lower) limit for the AP (resp. HT) PyC). We also use the same value for the interlayer distance $d_{002}=0.35 \mathrm{~nm}$. This value is typical for an AP PyC and slightly too high for the HT PyC, however, our point here is to focus on the structural differences induced by different nanotextures, everything else being equal. With these parameters we built initial atomistic configurations of 9926 atoms in a cubic box of $4.55 \mathrm{~nm}$ edge for the two small images and boxes of 18507 and 22198 atoms (cubes of 5.6 and $5.95 \mathrm{~nm}$ edges) for respectively the large 16 fringes AP and 17 fringes HT systems. Atoms were randomly inserted in the volumes with a non-overlap condition of $0.13 \mathrm{~nm}$ to ensure a reasonable initial energy.

As a sampling technique we chose an isothermal $N V T$ MD algorithm based on the stochastic thermostat proposed by Andersen [33]. In this method, Newton's equations of motion

$$
\dot{\mathbf{r}}_{i}=\mathbf{p}_{i} / m_{i}
$$

and

$$
\dot{\mathbf{p}}_{i}=-\nabla_{i} \mathcal{U}
$$


(where $\mathbf{r}_{i}, \mathbf{p}_{i}$ and $m_{i}$ are respectively the position, momentum and mass of particle $i, \mathcal{U}$ is the total potential energy defined in Eq. 2 and $\nabla_{i}$ indicates the derivative with respect to $\mathbf{r}_{i}$ ) are numerically integrated using the velocity Verlet algorithm [33] with a 0.2 fs timestep. After each step, every atom has a $0.2 \%$ probability to have its velocity redrawn from a Maxwell-Boltzmann distribution corresponding to the target temperature. The simulated annealing is performed by changing (lowering) this target temperature at every step according to a predefined cooling sequence.

Table 1: Summary of the different combinations of quench rates $(\partial T / \partial t)$ and $k_{I m}$ used in IGAR simulations performed on small (13 fringes) 3D image blocs (the total simulated time is also indicated).

\begin{tabular}{l|c|cccccccc|c}
\hline \hline \multirow{2}{*}{ Run type } & \multirow{2}{*}{$k_{I m}(\mathrm{eV})$} & \multicolumn{7}{|c|}{$\partial T / \partial t(K / p s)$} & at $T / 10^{3}=$ & \multirow{2}{*}{ Time (ps) } \\
& & $8-7$ & $7-6$ & $6-5$ & $5-4$ & $4-3$ & $3-2$ & $2-1$ & $1-0$ & \\
\hline A & 2 & 25 & 25 & 25 & 25 & 25 & 25 & 25 & 25 & 320 \\
B & 2 & 5 & 5 & 5 & 5 & 5 & 5 & 5 & 5 & 1600 \\
$\mathrm{C}$ & 2 & - & - & 2 & 2 & 2 & 2 & 2 & 2 & 3000 \\
$\mathrm{D}$ & 2 & 125 & 25 & 5 & 1 & 5 & 25 & 125 & 125 & 1504 \\
$\mathrm{E}$ & 1 & 5 & 5 & 5 & 5 & 5 & 5 & 5 & 5 & 1600 \\
$\mathrm{~F}$ & 4 & 5 & 5 & 5 & 5 & 5 & 5 & 5 & 5 & 1600 \\
\hline \hline
\end{tabular}

Three different values of $k_{I m}: 1,2$ and $4 \mathrm{eV}$, and different cooling sequences were tested on the small 13 fringes image blocks as summarized in table 1. 


\subsection{Structural analysis}

Once the IGAR simulation has been performed, and prior to structural analysis, a short (typically $100 \mathrm{ps)} \mathrm{MD} \mathrm{simulation} \mathrm{is} \mathrm{performed} \mathrm{at} 300 \mathrm{~K}$ using the AIREBO potential [34] to relax the structure (note that it is achieved only on the large atomistic models, not on the small systems used in section 3.2). This potential is a modification of the REBO potential including an adaptive treatment of van der Waals interactions between non-bonded atoms. Obviously, the image potential is switched off during this relaxation simulation. At that point, many structural properties of the resulting models are computed. It includes the usual average coordination numbers and bond angle distributions where two atoms form a bond if they are distant by less than $1.85 \AA$ (an intermediate value between first and second neighbours in graphene).

From that, ring statistics can be computed using the "shortest path ring" algorithm of Franzblau [35] where only threefold atoms are taken into account. To go a little further than the simple ring statistics, we have also developed a clustering approach to determine those threefold atoms belonging only to hexagonal rings (called pure $C_{6}$ atoms) and among them those atoms belonging to rings entirely made of pure $C_{6}$ atoms (noted pure $C_{6}$ rings). Finally, fragments, or clusters of rings, are defined on the basis that two pure $C_{6}$ rings sharing an atom belong to the same fragment. This algorithm is essentially the same as the one of Jain and Gubbins [36] except that only threefold atoms and hexagonal rings are considered.

HRTEM images of the PyC models are simulated using the multislice simulation approach as implemented in the NCEMSS software package [37]. In 
those simulations we adopted typical microscope parameters used to produce experimental images of PyC matrices: Philips CM30ST microscope with a $300 \mathrm{kV}$ accelerating voltage, Scherzer defocus of $-58 \mathrm{~nm}$, spread of defocus of $7 \mathrm{~nm}$, spherical aberration $\left(C_{s}\right)$ of $1.2 \mathrm{~mm}$, convergence angle of $0.5 \mathrm{mrad}$ and aperture radius of $4 \mathrm{~nm}^{-1}$.

Finally, the reduced pair distribution functions (PDFs), defined as

$$
G(r)=4 \pi \rho r[g(r)-1]
$$

where $\rho$ is the atomic density and

$$
g(r)=\frac{1}{4 \pi r^{2} \rho N}\left\langle\sum_{i=1}^{N} \sum_{j \neq i}^{N} \delta\left(r-r_{i j}\right)\right\rangle
$$

is the atomic pair distribution function (where $N$ is the number of atoms and $\delta\left(r-r_{i j}\right)=1$ when atoms $i$ and $j$ are distant by $r$ and 0 otherwise), were computed from a statistical averaging on an equilibrated MD simulation at $300 \mathrm{~K}$.

\section{Results}

\subsection{Synthetic 3D HRTEM-like images}

The experimental HRTEM images of the AP (Fig. 2a) and HT (Fig. 2b) PyCs are shown after high and low frequency filtering on Figs $2 \mathrm{c}$ and $2 \mathrm{~d}$ respectively. As compared to the raw images, we can see that the filtered images only contain the fringe characteristics, namely, their length, curvature, contrast, etc ... More specifically, we see that fringes of the AP PyC are neatly shorter, more curved and more interconnected than those of the HT PyC. 

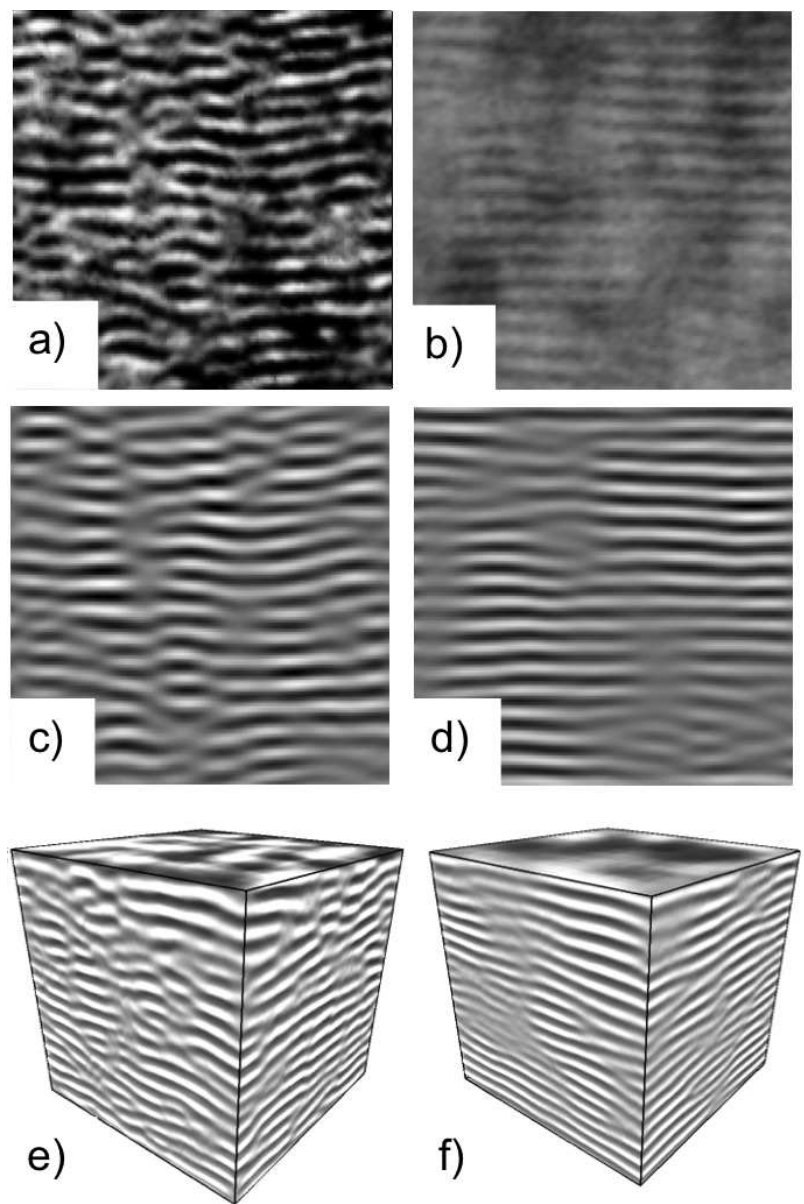

Figure 2: Illustration of the 3D HRTEM-like images synthesis. Experimental HRTEM image of a PyC as prepared (AP)(a) and heat treated (HT)(b); Filtered HRTEM image of a PyC AP (c) and HT (d); Synthesized 3D HRTEM-like image of a PyC AP (e) and HT (f). Images (a, c and e) counts on average 16 fringes in the stacking direction against 17 fringes for images (b, d and $\mathrm{f})$. 
$3 \mathrm{D}$ views of the synthesis results are shown in Fig. 2e and f. Perceptually speaking, the two solid textures are quite convincing. They are free from major artifacts. 2D slices of these solid textures show morphological properties close to the ones observed in the corresponding filtered HRTEM images: straight and distorted fringe lengths and curvatures are similar. The overall anisotropy seems to be respected as discussed in [28]. Fringe lengths and tortuosities calculations would allow for a more thorough comparison, however the size of the synthesized blocks, only of around $6 \mathrm{~nm}$, does not allow yet for such a study; this will be achieved in the future on larger blocks.

\subsection{Preliminary study on a 13 fringes $A P P y C$}

We start the IGAR study by searching for the best cooling sequence and the best value of $k_{I m}$ using a 13 fringes image block synthesized from the HRTEM image of an AP PyC. This preliminary study is presented in more details in the first section of the supporting material and we only discuss here the main results. The final properties $\left(\mathcal{U}_{R E B O}, \mathcal{U}_{H R T E M}\right.$, coordination data and ring statistics) of models constructed using IGAR simulations with the six sets of parameters of table 1 are summarized in table 2 .

We can see in table 2 that, among the six model constructions, run D is the one that results in the lowest value of $\mathcal{U}_{R E B O}$, the largest $s p^{2}$ fraction and the largest amount of six-membered rings, while keeping a low value of $\mathcal{U}_{H R T E M} / k_{\text {Im }}$. It is thus the best combination of quench algorithm and $k_{I m}$. Note the particular interest of the ramp cooling sequence, which performs better than a uniform cooling at $2 \mathrm{~K} / \mathrm{ps}$ with the same value of $k_{I m}$ despite being twice shorter in terms of computer time. As a consequence, IGAR parameters of run D will be used in what follows to build large AP and HT 
Table 2: Final properties of 13 fringes AP PyC models.

\begin{tabular}{l|c|c|c|c|c|c}
\hline \hline Run & $\mathrm{A}$ & $\mathrm{B}$ & $\mathrm{C}$ & $\mathrm{D}$ & $\mathrm{E}$ & $\mathrm{F}$ \\
\hline$k_{\text {Im }}(\mathrm{eV})$ & 2 & 2 & 2 & 2 & 1 & 4 \\
\hline Quench rate $(\mathrm{K} / \mathrm{ps})$ & 25 & 5 & 2 & $\mathrm{ramp}$ & 5 & 5 \\
\hline $\mathcal{U}_{R E B O}(\mathrm{eV})$ & -7.132 & -7.209 & -7.236 & -7.239 & -7.200 & -7.192 \\
$\mathcal{U}_{H R T E M} / k_{\text {Im }}$ & 0.307 & 0.305 & 0.305 & 0.305 & 0.322 & 0.294 \\
\hline$s p$ fraction & 0.007 & 0.004 & 0.003 & 0.003 & 0.002 & 0.007 \\
$s p^{2}$ fraction & 0.951 & 0.965 & 0.970 & 0.970 & 0.961 & 0.966 \\
$s p^{3}$ fraction & 0.042 & 0.031 & 0.027 & 0.027 & 0.037 & 0.027 \\
\hline$C_{5}$ fraction & 0.142 & 0.093 & 0.069 & 0.058 & 0.109 & 0.092 \\
$C_{6}$ fraction & 0.689 & 0.801 & 0.852 & 0.875 & 0.755 & 0.806 \\
$C_{7}$ fraction & 0.143 & 0.100 & 0.077 & 0.063 & 0.122 & 0.094 \\
$C_{8}$ fraction & 0.015 & 0.004 & 0.002 & 0.004 & 0.011 & 0.007 \\
\hline \hline
\end{tabular}


PyC models.

\subsection{Atomistic pyrocarbon models}

\subsubsection{General structural features}

The IGAR procedure is now applied to the 16 fringes image block of the AP PyC (Fig. 2e) and to the 17 fringes image block of the HT PyC (Fig. 2f). Snapshots of the models obtained for these two materials, after a short MD relaxation at $300 \mathrm{~K}$ using the AIREBO potential, are shown in respectively Fig. 3 and Fig. 4. Their main structural parameters are given in table 3 (a movie, illustrating the construction of the $\mathrm{AP} \mathrm{PyC}$ model is also given as supporting material, section 2).

Confirming the studies on smaller systems presented earlier, both models are typical of $s p^{2}$ graphitic carbons (mostly 3 -fold $\mathrm{C}$ atoms and $C_{6}$ rings). They also contain similar amounts of pentagonal and heptagonal rings, 5-6 $\%$ for both models and a few octagonal rings (0.2-0.3 \%). Also, in agreement with the high fractions of hexagonal rings, the fractions of pure $C_{6}$ atoms $\left(f_{p C_{6}}^{a t o m}\right)$ and of pure $C_{6}$ rings $\left(f_{p C_{6}}^{r i n g s}\right)$ are high for both models. Some years ago Vallerot et al. [5] have reported on angle resolved electron energy loss spectroscopy (EELS) experiments to assess the hybridization of PyC matrices. Their results indicate that RL PyCs contain $82.5 \%$ of pure $s p^{2}$ carbons, the remaining atoms having, according to them, a $s p^{2+\varepsilon}$ hybridization, due to out-of-plane distorsions as well as some $C_{7} / C_{5}$ rings. They also claim than $s p^{3}$ hybridization should be very small in these materials as confirmed by the very weak Raman $\mathrm{D}$ band and by the fact that a significant amount of $s p^{3}$ carbon atoms in such low nanoporosity materials would be incompatible with their densities of around $2.1 \mathrm{~g} / \mathrm{cm}^{3}$. Our models comprising 97 and $99 \%$ of 


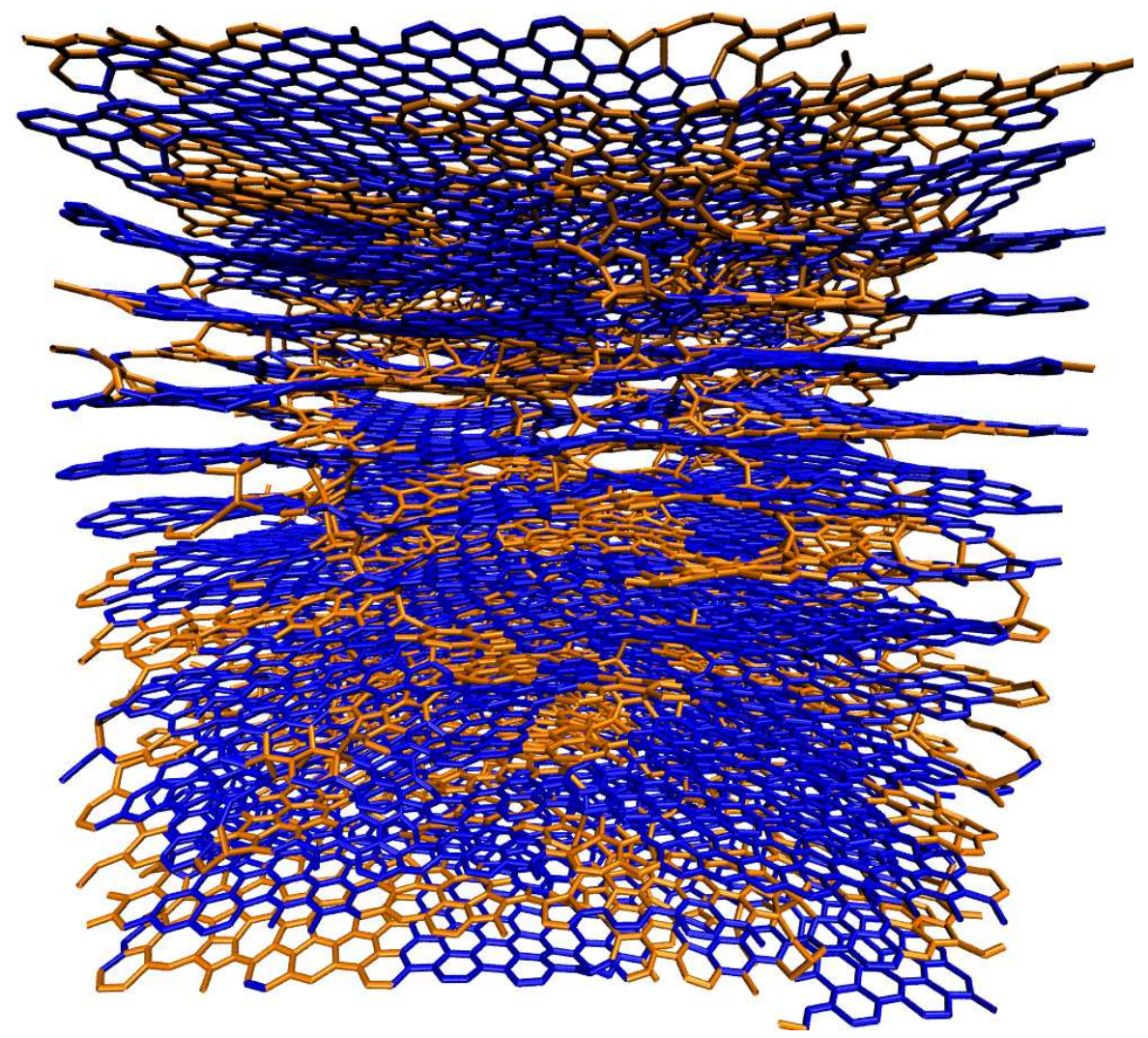

Figure 3: Snapshot of the 16 fringes $\mathrm{AP} \mathrm{PyC}$ model. Bonds between carbon atoms belonging to pure hexagonal domains are shown with blue sticks. Other $\mathrm{C}-\mathrm{C}$ bonds are shown with orange sticks. 


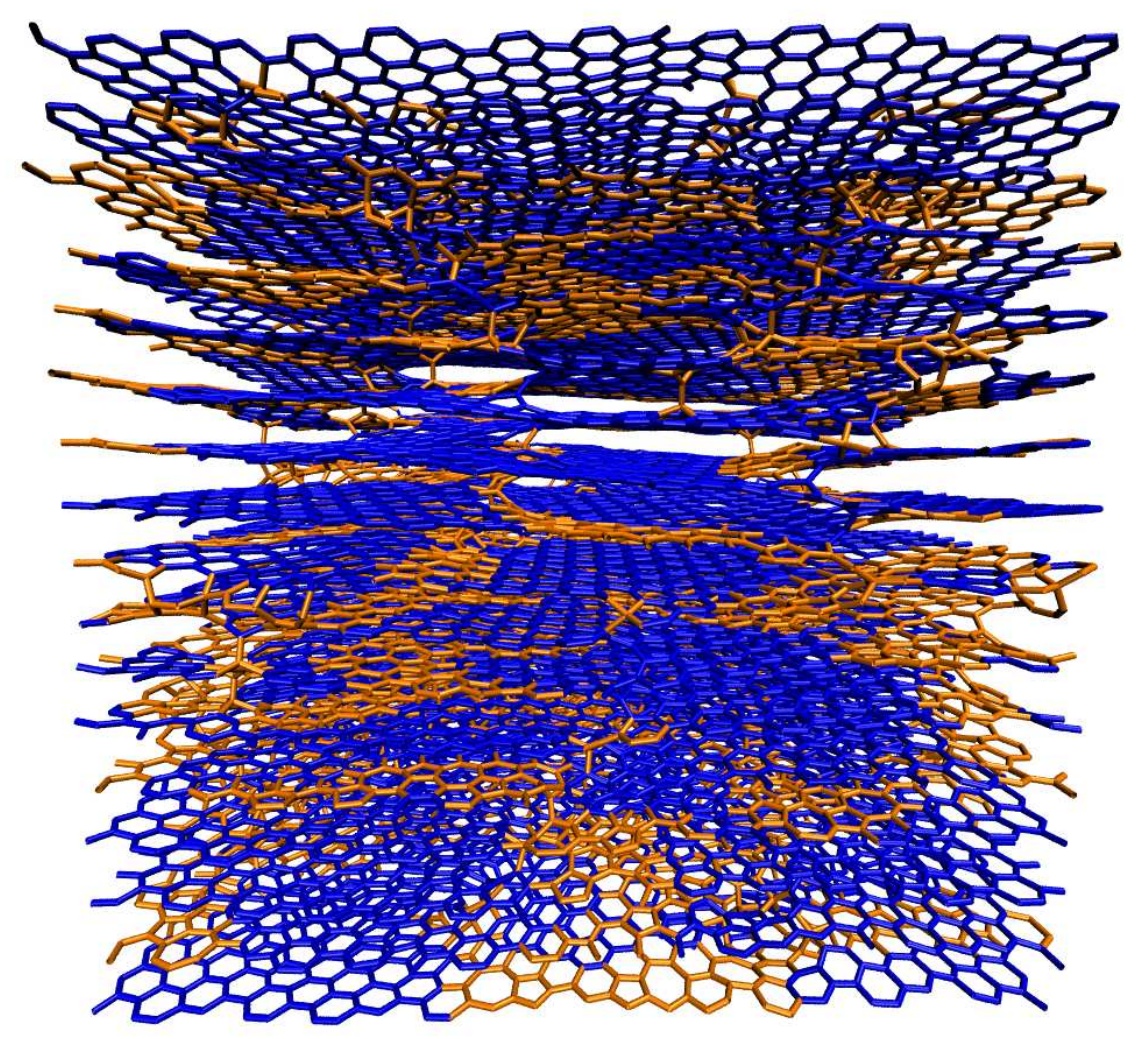

Figure 4: Same as Fig. 3 for the 17 fringes HT model. 
Table 3: Final properties of PyC models constructed from a 16 fringes AP PyC image and a 17 fringes HT PyC image (a 2 $\mathrm{eV}$ value of $k_{I m}$ was used with the ramp cooling algorithm).

\begin{tabular}{l|c|ccc|cccc|cc}
\hline \hline Material & $U_{A I R E B O}$ & $f_{s p}$ & $f_{s p^{2}}$ & $f_{s p^{3}}$ & $f_{C_{5}}$ & $f_{C_{6}}$ & $f_{C_{7}}$ & $f_{C_{8}}$ & $f_{p C_{6}}^{\text {atom }}$ & $f_{p C_{6}}^{\text {ring }}$ \\
\hline $\mathrm{AP}$ & -7.283 & 0.005 & 0.973 & 0.022 & 0.054 & 0.886 & 0.057 & 0.003 & 0.753 & 0.700 \\
$\mathrm{HT}$ & -7.300 & 0.002 & 0.990 & 0.008 & 0.054 & 0.889 & 0.055 & 0.002 & 0.764 & 0.737 \\
\hline \hline
\end{tabular}


threefold atoms for respectively the AP and HT PyCs are totally compatible with the latter assumption. Moreover, the fractions of pure $C_{6}$ atoms (respectively 75 and $76 \%$ for the $\mathrm{AP}$ and $\mathrm{HT}$ PyCs) and pure $C_{6}$ rings (70 \% for AP, $74 \%$ for HT) are not far from the $82.5 \%$ pure $s p^{2}$ atoms measured by these authors and our models suggest that the " $s p^{2+\varepsilon " ~ h y b r i d i z a t i o n ~ w o u l d ~}$ actually essentially be due to non-hexagonal rings.

Even though the presented coordination and ring statistics are interesting and compatible with experimental observations, these properties, mainly characterizing short-range order in the materials, are unable to unambiguously discriminate between the AP and HT PyCs. Nevertheless, and as expected for pyrocarbons, they differ considerably from data reported for atomistic models of more disordered carbons like chars [38], saccharose based carbons $[17,36]$ and glassy carbon [18], constructed using HRMC methods. Indeed, those models show much lower $s p^{2}$ contents (between 50 and $90 \%$ ) and fractions of hexagonal rings.

The bond angle distributions shown in Fig. 5 show two very similar curves, almost indistinguishable from each other, and narrowly peaked around the $120^{\circ}$ angle of the honeycomb lattice for both the AP and HT models. They are only slightly broader than the corresponding distribution for hexagonal graphite due to the existence of $s p^{3}$ carbon atoms and $C_{5}$ rings (low angle broadening) as well as $C_{7}$ and $C_{8}$ rings (large angle broadening). Again, bond angle distributions reported for atomistic models of more disordered carbons $[18,38]$ are much broader than the ones reported here for PyCs.

As has been quantified, the two models are essentially the same at very short range, typically at the length scale of one (or a few) chemical bond(s). 


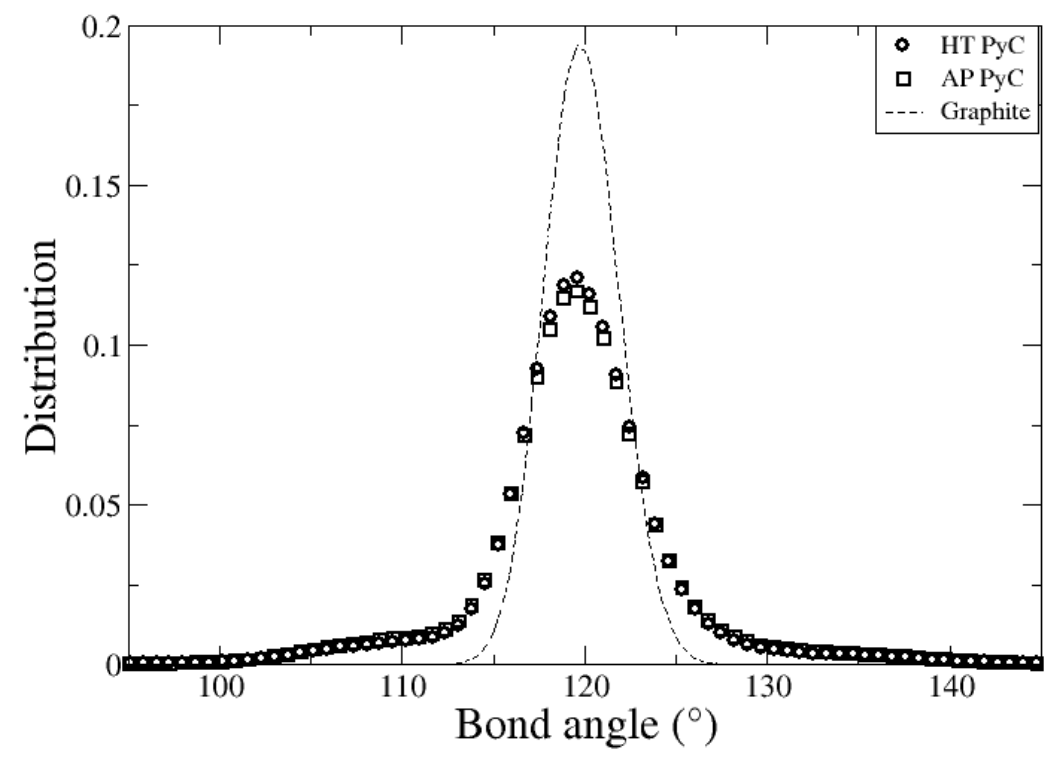

Figure 5: Room temperature bond angle distributions of the 16 fringes AP model (squares), the 17 fringes HT model (circles) and hexagonal graphite (dotted line) as obtained from MD simulations. 
Moreover, looking at Fig. 3 and 4, we see that they are both made of some nanosized pure hexagonal domains made of pure $C_{6}$ rings (blue), connected together by defect containing domains (orange). However, these figures also clearly highlight some differences in their nanotextures, as discussed hereafter.

\subsubsection{Out-of-plane defects features}

Looking at Fig. 4 we can see that the HT model is characterized by extended, and rather flat, carbon sheets, with few interconnections. The AP model (see Fig. 3), on the other hand seems to present smaller and more curved sheets with numerous interconnections along the stacking direction. These differences are clearly confirmed in Figure 6, showing 2-nm thick slices of the models, taken along the stacking direction. Indeed, in the case of the $\mathrm{HT} \mathrm{PyC}$ (Fig. 6b) some well defined flat domains, extending on the whole width of the simulation box $(5.95 \mathrm{~nm})$, are neatly visible; in the AP PyC model (Fig. 6a), such domains roughly never exceed $2 \mathrm{~nm}$.

Fig. 7 shows a chunk of the AP model, highlighting the screw dislocation structure of this material. As can be seen, those domains remind us of the shape of the access ramp in multi-floors car parks. Moreover, as shown in the hexagon preserving carbon nanofoams predicted some years ago by Kuc and Seifert using density functional tight binding calculations [39], these dislocations can be well accommodated by pure hexagonal $s p^{2}$ carbon structures; this is almost the case of the dislocation appearing on the top right corner of Fig. 7. Such screw dislocations are also found in the HT model, yet in much lower amount. 

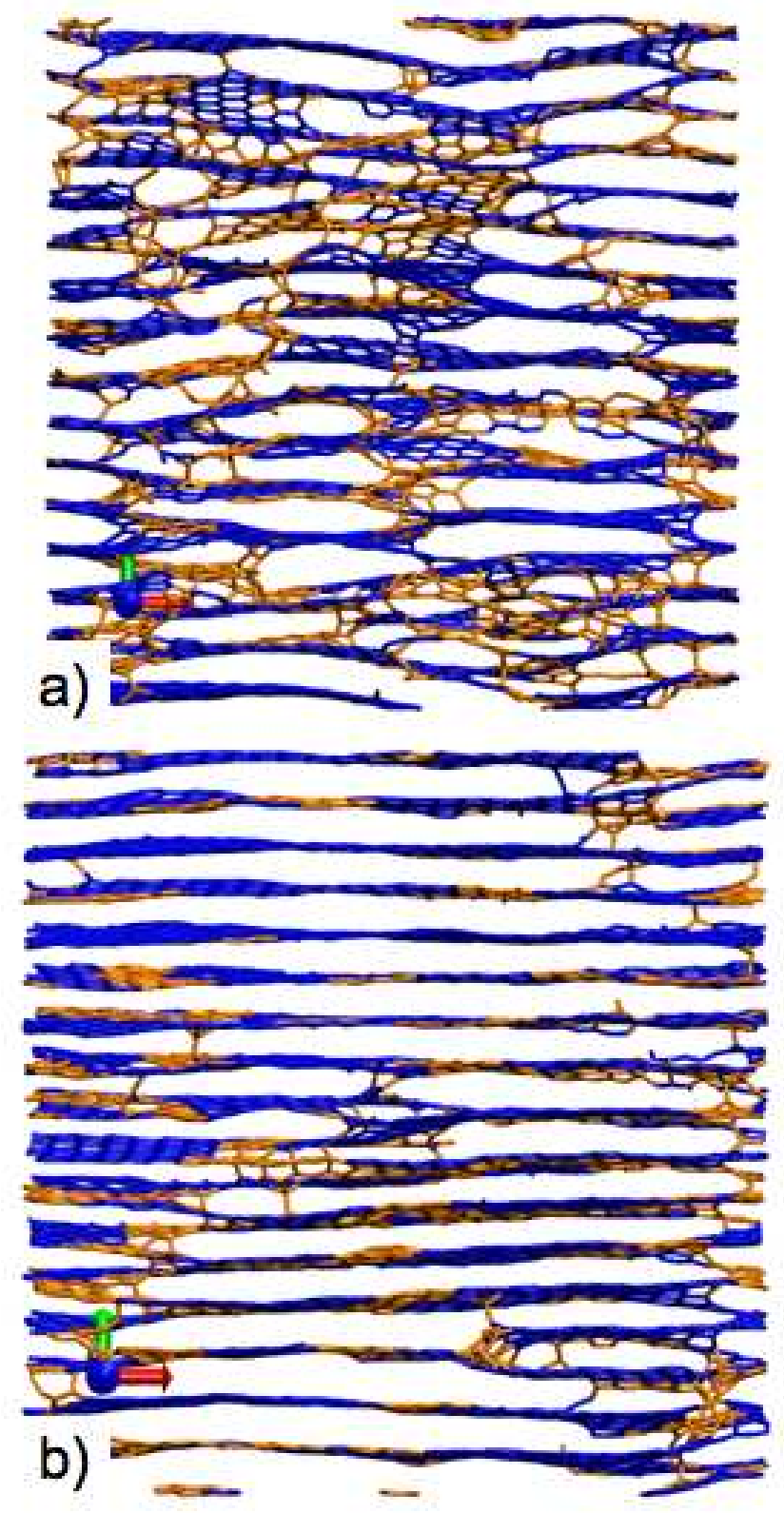

Figure 6: 2 nm-thick slices of the AP (a) and HT (b) models (same color code as in Figures 3 and 4$)$. 


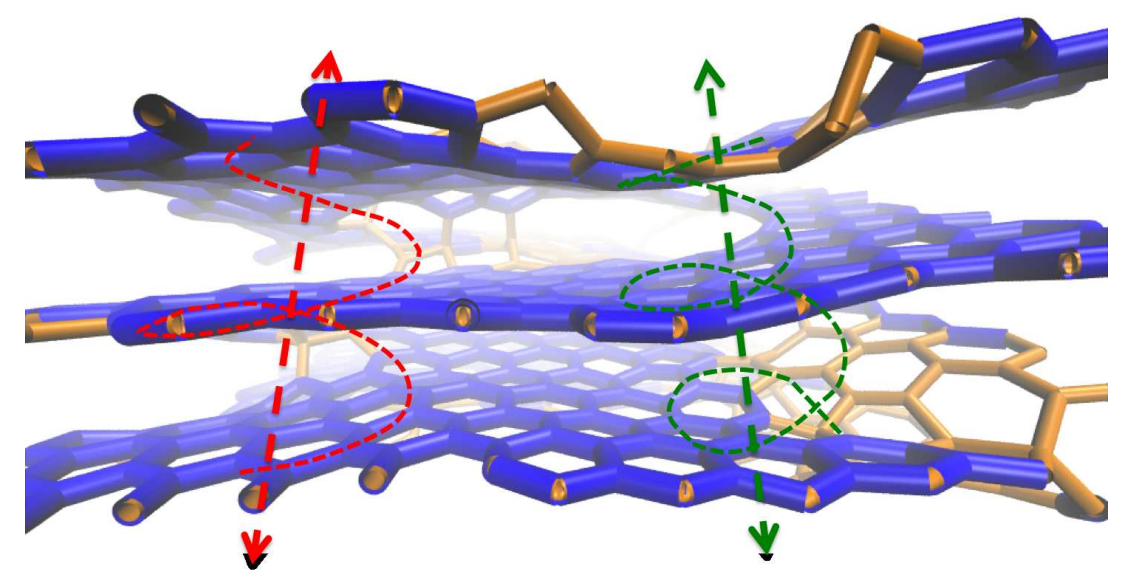

Figure 7: Chunk of the AP PyC model showing a screw dislocation pair (red and green dashed lines and arrows; chemical bonds are displayed with the same color code as in Fig. 3 and 4$)$.

\subsubsection{In-plane defects features}

We show in Fig. 8 two carbon sheets taken from the models. Common to the two models, and as already said below, the sheets contain nanosized domains entirely made of $s p^{2}$ pure $C_{6}$ rings (in blue). These domains are connected together by disordered areas (orange) and by screw dislocations (holes). More precisely, the disordered areas, in agreement with data from table 3 , are essentially made of $C_{5}, C_{7}$ and "non pure" $C_{6}$ rings (i.e. $C_{6}$ rings containing at least an atom that is not threefold or that also belongs to another kind of ring) as well as a few fourfold or twofold atoms and $C_{8}$ rings. Nevertheless, those areas are not as "disordered" as we could think at first sight. Indeed, as shown with red lines on Fig. 8, $C_{5}$ and $C_{7}$ rings are most of the time grouped by pentagon-heptagon pairs $\left(C_{5} / C_{7}\right.$ pairs $)$ and 


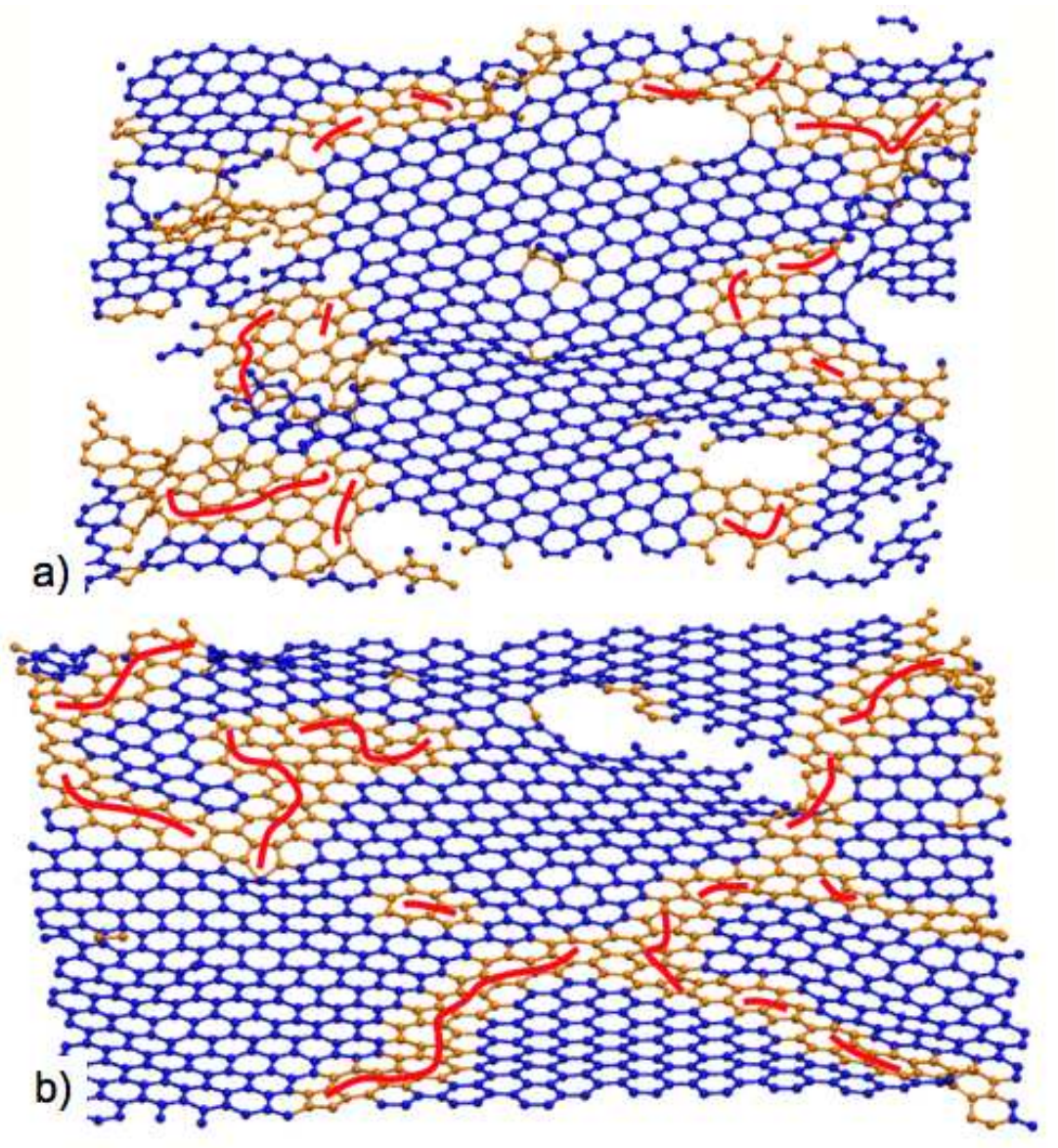

Figure 8: Carbon sheets (0.3 nm-thick in-plane slices) of the AP (a) and HT (b) PyC models (same color code as in Figures 3 and 4; red lines indicate the pentagon/heptagon pairs and lines of pairs). 
lines or networks of $C_{5} / C_{7}$ pairs. This is in agreement with recent theoretical works on non-hexagonal ring defects in graphene, showing the high stability of defects based on the $C_{5} / C_{7}$ pattern [40-42]. Also, it is important to note that we did not observe any isolated pentagon, a defect associated to highly curved structures like fullerenes, glassy carbons or other non graphitizing carbons $[43,44]$. Comparing now Fig. 8a and b allows confirming some previous remarks, namely that the AP model presents more dislocations and more wavy sheets than the HT model. Also, as we can see in these figures, if the AP model shows only isolated $C_{5} / C_{7}$ pairs or very short $C_{5} / C_{7}$ lines, the HT model on the opposite shows rather extended lines, made of up to six or seven $C_{5} / C_{7}$ pairs.

In order to understand a little further the role played by these disordered domains, and their origin, we show in Fig. 9 three portions of carbon sheets taken from the HT model. As we can see in the three panels of this figure, these extended $C_{5} / C_{7}$ lines actually act as grain boundaries between disoriented hexagonal domains. We can guess that the hexagonal domains form first and that the networks of pentagons and heptagons develop themselves when these hexagonal domains with different "in-plane" orientations merge. Also, a few interesting features can be observed on these figures. First, as highlighted by a green circle, a Stone-Thrower-Wales defect is clearly visible in Fig. 9a. Second, the disordered area of Fig. 9b, almost circular, surrounds a very small hexagonal domain with a different orientation than the rest of the sheet. Finally, the pentagon and heptagon containing area delimited by the green rectangle on Fig. 9c is particularly interesting. Indeed, this domain is space-filling, meaning that entire sheets can be made out of its repetition 


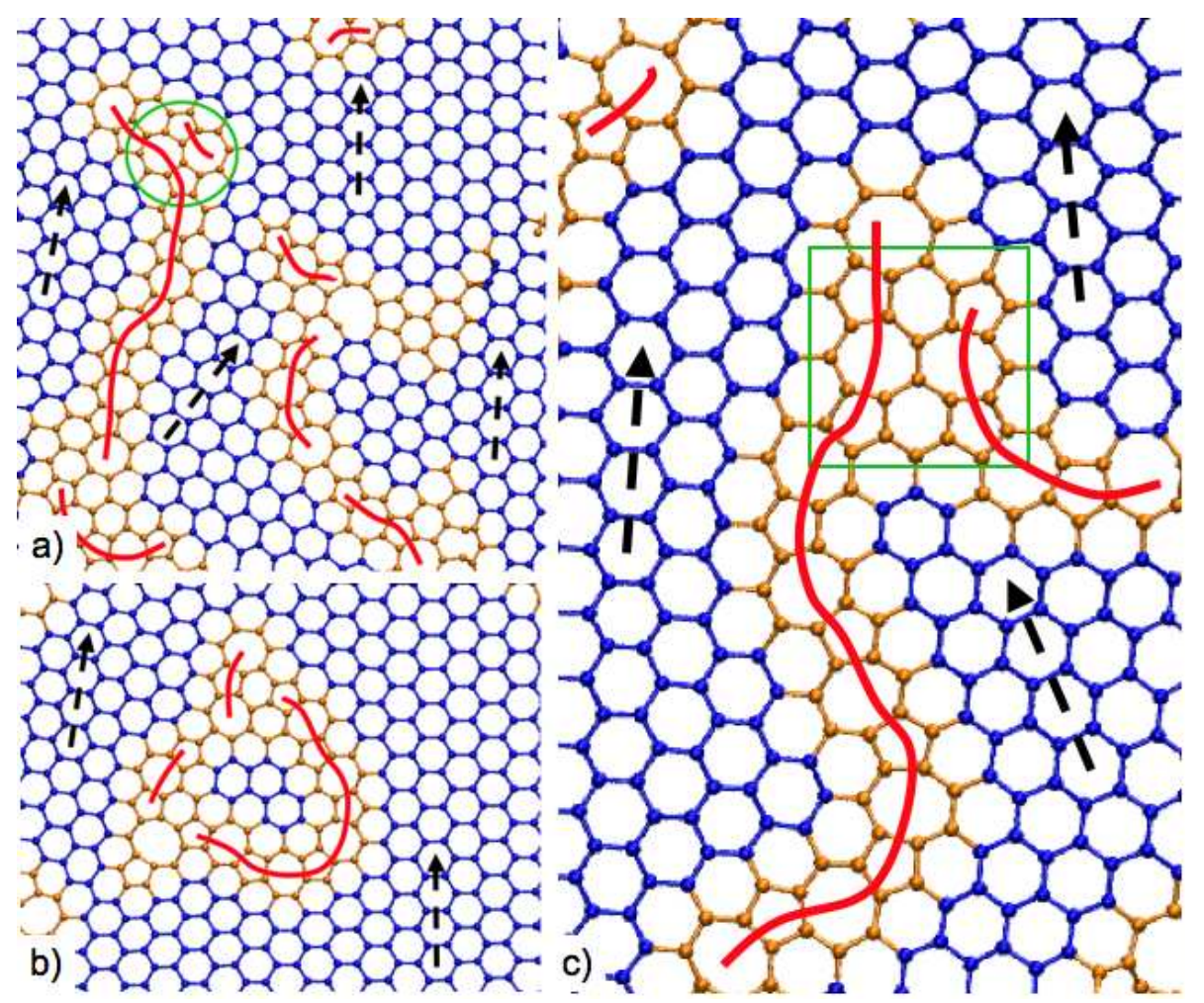

Figure 9: Snapshots of some carbon sheets taken from the HT model (same color code as in Fig. 8; orientations of some hexagonal domains are indicated by dashed black arrows, a Stone-Thrower-Wales defect and a $C_{5}^{2} C_{6}^{3} C_{7}^{2}$ defect, studied in details elsewhere [45], are respectively highlighted by a green circle (a) and a green rectangle (c)). 
by translation. The structure and properties of nanocarbons based on it (sheets and tubes) have been studied elsewhere [45].

In an attempt to quantify the structural differences between the two materials at the mesoscale we have performed a cluster analysis of pure $C_{6}$ rings. Fig. 10 gives histograms of the different fragments (clusters of connected pure $C_{6}$ rings) found for these models. As can be seen on the upper panel of Fig. 10, the AP model counts much more very small fragments than the HT one, for instance, 17 isolated pure $C_{6}$ rings are found in the AP model against 3 in the HT model (note that according to our definition, an isolated pure $C_{6}$ ring actually means a $C_{6}$ ring surrounded by six non pure $C_{6}$ rings). The structure of the AP Pyc can actually be discribed by a combination of very small "mono-sheet" fragments of less than 80 pure $C_{6}$ rings and two very large "multi-sheets" fragments counting for more than $90 \%$ of the pure $C_{6}$ rings present in the model which indicates a very high density of screw dislocations (a graphite sample of similar dimensions would be made of grephene sheets of only 620 rings). On the opposite, the $\mathrm{HT} \mathrm{PyC}$ is made of larger mono-sheet fragments, indicating a better "in-plane" order, and smaller "multi-sheet" fragments, indicating a lower density of dislocations.

\subsubsection{HRTEM validation}

In order to verify that our models actually corresponds to the experimental materials we now compare the experimental HRTEM images to those simulated from the models using the multi-slice method [37]. Fig. 11 shows the experimental HRTEM images of AP (Fig. 11a) and HT (Fig. 11b) PyCs after high and low frequency filtering (identical to Figs 2c and 2d), together with the images simulated from the AP (Fig. 11c) and HT (Fig. 11d) atom- 


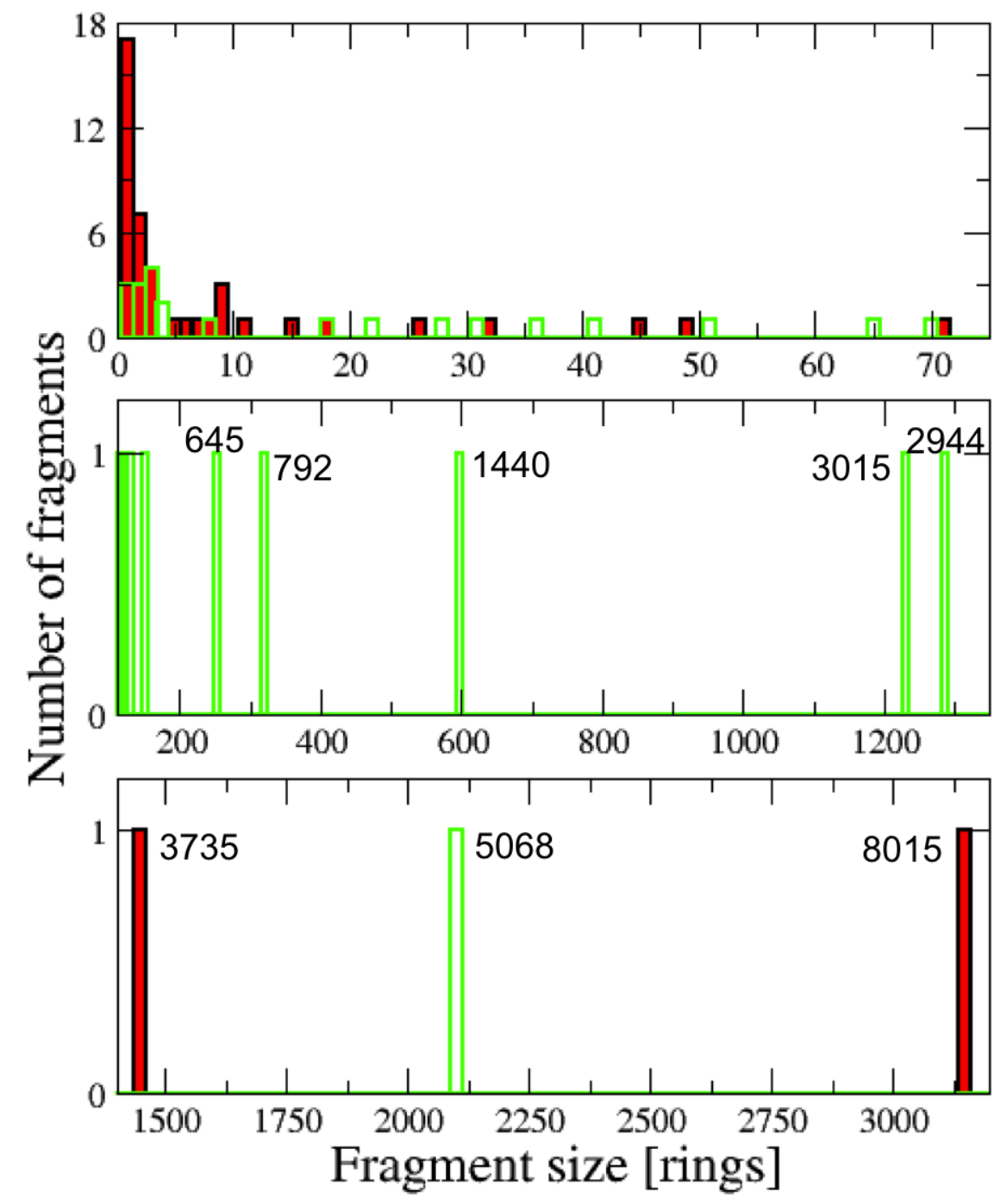

Figure 10: Histogram of the number of fragments found in the AP (red filled bars) and the HT (green empty bars) models with respect to their size (in terms of the number of pure $C_{6}$ rings (the histogram is split into three panels according to the fragment size; small fragments: top, large fragments: bottom; numbers in the panels indicate the fragments sizes in atoms). 

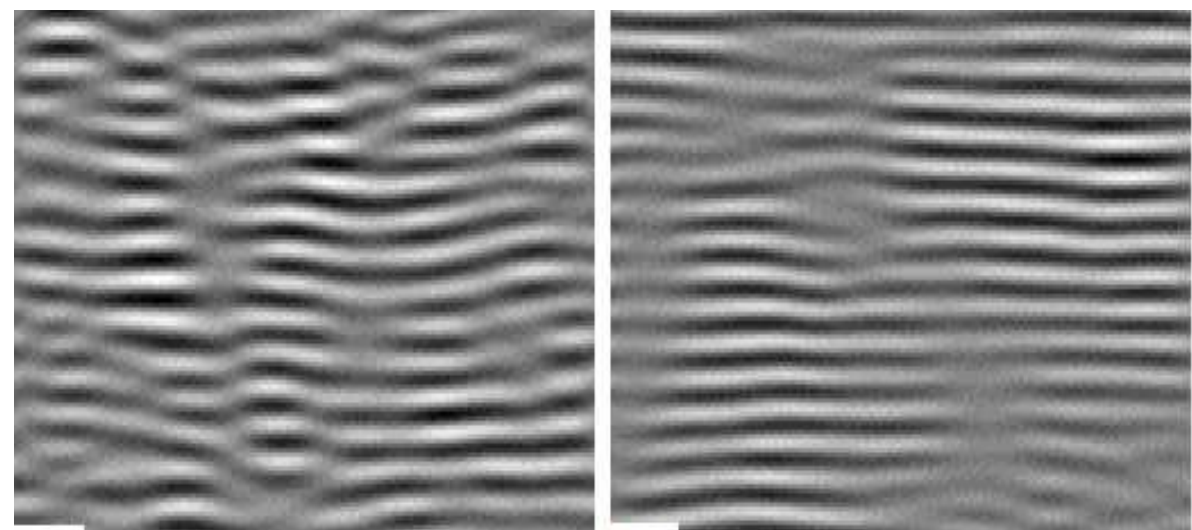

a)

b)

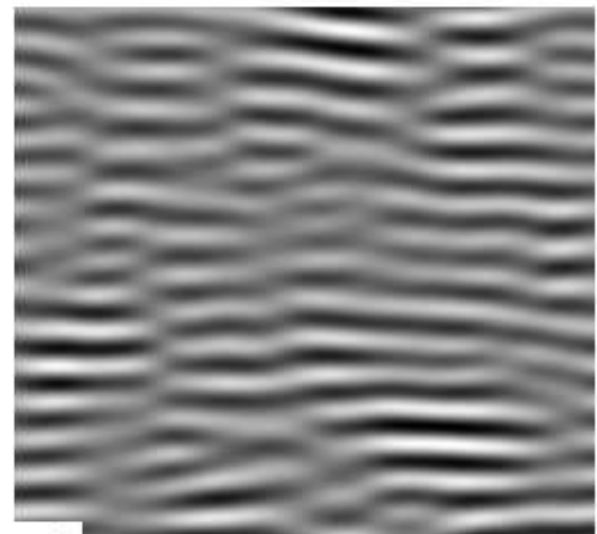

c)

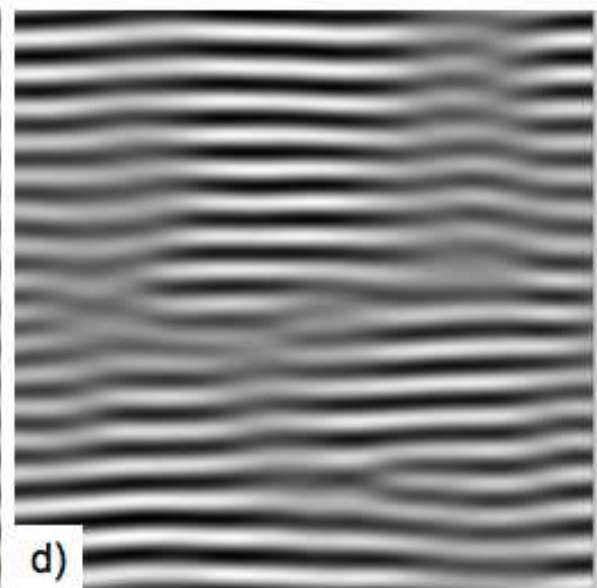

Figure 11: Comparison between experimental and simulated HRTEM images. a: filtered experimental image of the $\mathrm{AP} \mathrm{PyC}$ (equivalent to Fig. 2c); b: filtered experimental image of the HT PyC (equivalent to Fig. 2d); c: simulated image from the AP model; d: simulated image from the HT model. 
istic models.

Bearing in mind that the 3D image blocks guiding the IGAR simulations are not directly inferred from 2D images but from statistical descriptors of these images, images simulated from the model do not have to be identical replicas of experimental images but statistically equivalent to them (especially second order statistics, describing the fringes properties, while first order statistics are more sensitive to the parameters governing the virtual microscope in the image simulation process). Looking at Fig. 11 we can see that apart from differences in contrast, the similarity in terms of fringes undulations and junctions, between initial images (Fig. 11a and 11b) and their corresponding simulated images (Fig. 11c and 11d) is obvious and confirm that the atomistic models contain most of the nanotextural information present in the HRTEM images.

Fig. 12 presents the gradient orientation maps computed from the HRTEM images of Fig. 11. In such an image, a red pixel indicates a zone with a vertical gradient, $i$. e. a horizontal fringe characterizing a graphitic order, and yellow/blue pixels correspond to areas with horizontal gradients (defective domains/stacking faults). Comparing Fig. 12a to 12c confirms that the experimental (Fig. 11a) and simulated (Fig. 11c) HRTEM images of the AP material present similar defects, both in terms of density and extent. A similar observation can be made for the HT material (see the good match between Fig. 12b and d). Obviously, images from the AP PyC (Fig. 12a and c) show higher amounts of defects (and more extended defects) than the HT images (Fig. 12b and d). Also, it is interesting to notice the close similarities between the fringes observed for the AP PyC (Fig. 11a or 11c) and the bond 

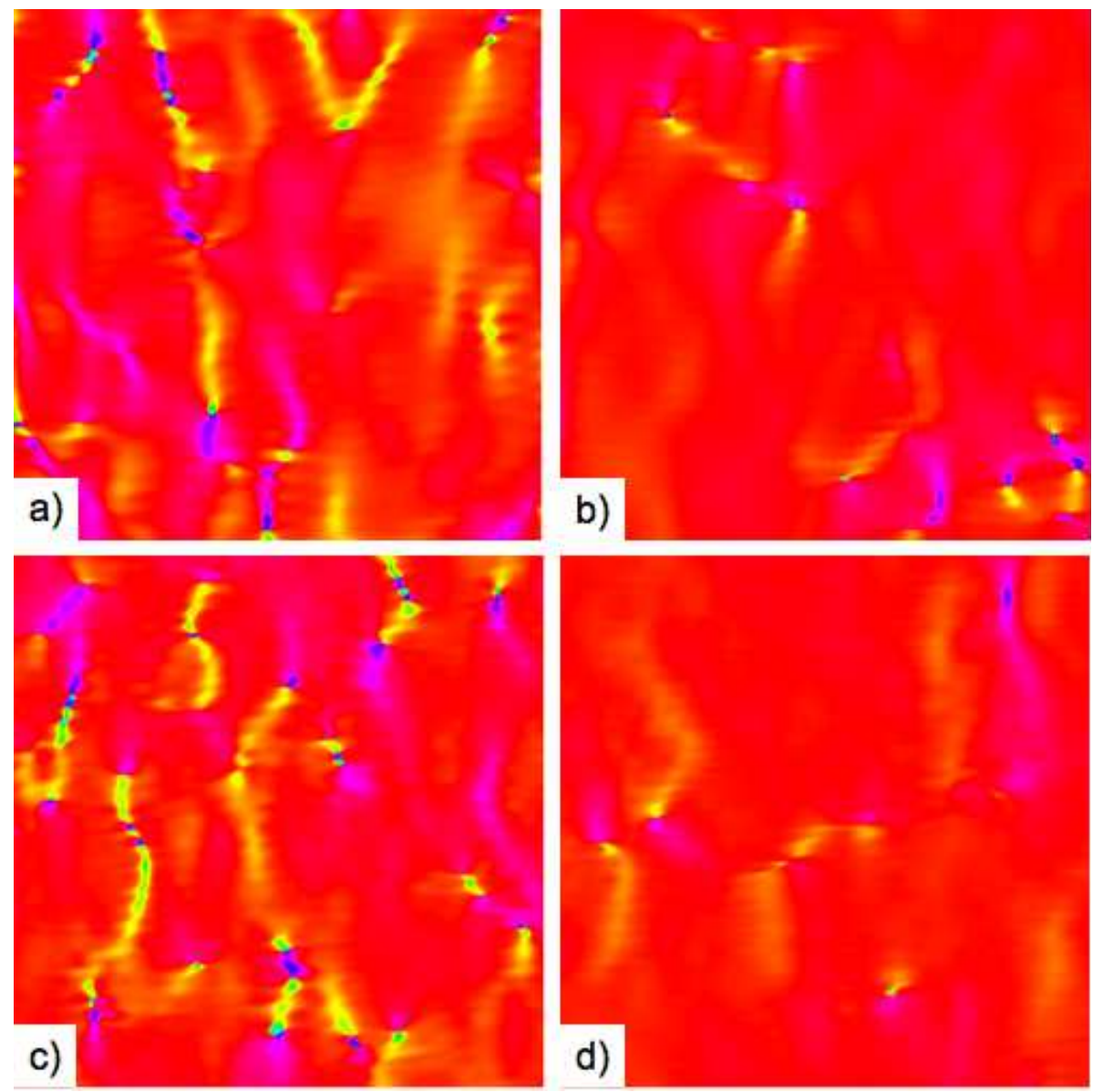

Figure 12: Orientation maps of PyCs HRTEM images. a, b, c and d respectively show maps of the gradient orientations of Fig. 11a, b, c and d; red: vertical gradient, blue or yellow: horizontal gradient. 
network of the AP model (Fig. 6a) on one side, and between the fringes of the HT PyC (Fig. 11b or 11d) and the bond network of the HT model (Fig. $6 \mathrm{~b})$ on the other side. For instance, the many screw dislocations observed in the atomistic model of the AP PyC (see Fig. 6a) seem to correspond pretty well to the blurred domains of the simulated HRTEM image for this model (Fig. 11c) or the defects observed on the corresponding orientation map (Fig. 12c). This can be also visualized in the supporting material movie (S2).

\subsubsection{Pair distribution functions}

We plot in Fig. 13 the reduced pair distribution functions computed in the range $[0: 30 \AA]$ for the AP and HT models (the functions are split into three panels according to the interatomic distance, for clarity). Very recently, we have been able to prepare a bulk sample of rough laminar pyrocarbon. Its reduced $\mathrm{PDF}, G(r)$, measured using neutron powder diffraction, is also shown in Fig. 13. All details regarding this material can be found in a recent publication [10]. We add that it resides at the high anisotropy and low defect corner of the RL PyC domain in the Raman-based classification of Bourrat et al. [7] and that its nanotexture, as observed in HRTEM, seems to be intermediate between the ones of the AP and HT PyCs.

We can see in Fig. 13 that every experimental peak is present in both the AP and HT models whatever the interatomic distance $r$ and apart from unphysical oscillations due to the Fourier transform of $S(Q)$ (see the experimental function between 0 and $1.3 \AA$ ). The superimposition of model and experimental PDFs is especially impressive for short interatomic distances (top panel) where only slight differences can be noted: (i) the model PDFs show thinner peaks than the experimental one up to $5 \AA$ and (ii) the HT 

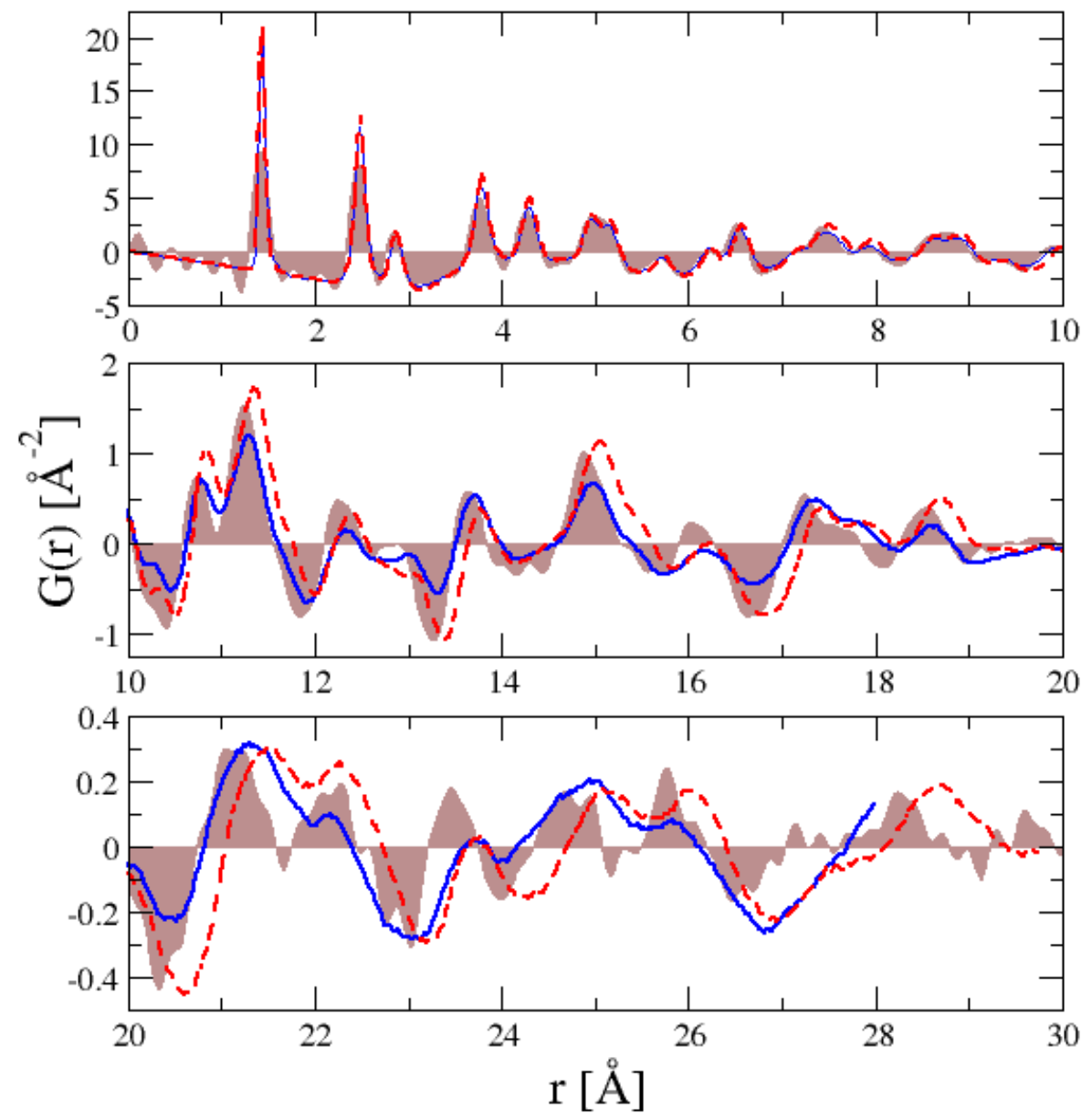

Figure 13: Reduced pair distribution function $(G(r))$ of the AP (solid blue line) and HT (dashed red line) PyC models. The experimental $G(r)$ of a rough laminar PyC (PyC-1 in Ref [10]) is also shown as a filled curve for comparison (note that the functions are split

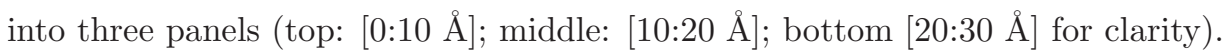


model has very slightly thinner peaks than the AP model. Moving to the central panel of Fig. 13 confirms the previous story though unraveling that peak locations seem to be a little shifted (actually, scaled, as we will see) to higher distances when comparing model PDFs to the experimental one, peaks of the HT model being the most shifted ones. Looking more closely at short $r$ values, we find first neighbors peaks at respectively 1.42, 1.44 and $1.44 \AA$ for the experimental function, the AP model and the HT model. Second neighbors peaks appear at 2.46, 2.47 and $2.47 \AA$ (same order) and third neighbors peaks at 2.83, 2.86 and $2.87 \AA$. Scaling $r$ values of the model PDFs by respectively 0.995 and 0.989 actually allows for an almost perfect match of the peaks locations on the whole $r$ range (see the third section of the supported material for an "r-scaled" version of Fig. 13).

The too low thicknesses of the peaks at short $r$ together with their shifts to larger $r$ at long distances are typical signatures of tensile stress in the material. Computing the stress tensors of the relaxed models we indeed find tensile in-plane stresses of around 22 and $26 \mathrm{GPa}$ for respectively the AP and HT models (full stress tensors are given in the fourth section of the supporting material). These stresses are actually artefact due to the use of the AIREBO potential. Indeed this potential underestimate the carbon-carbon bond length in aromatic rings and simulation of graphite at experimental density give rise to a similar tensile stress with 17 GPa in-plane components. Computing the model's properties with this potential actually leads to computing the properties of stressed PyCs. Using the REBO potential would avoid this artefact but in this latter case many-properties would be unreasonnable, especially those influenced by out-of-plane vibrations, due to the 
absence of van der Waals interactions.
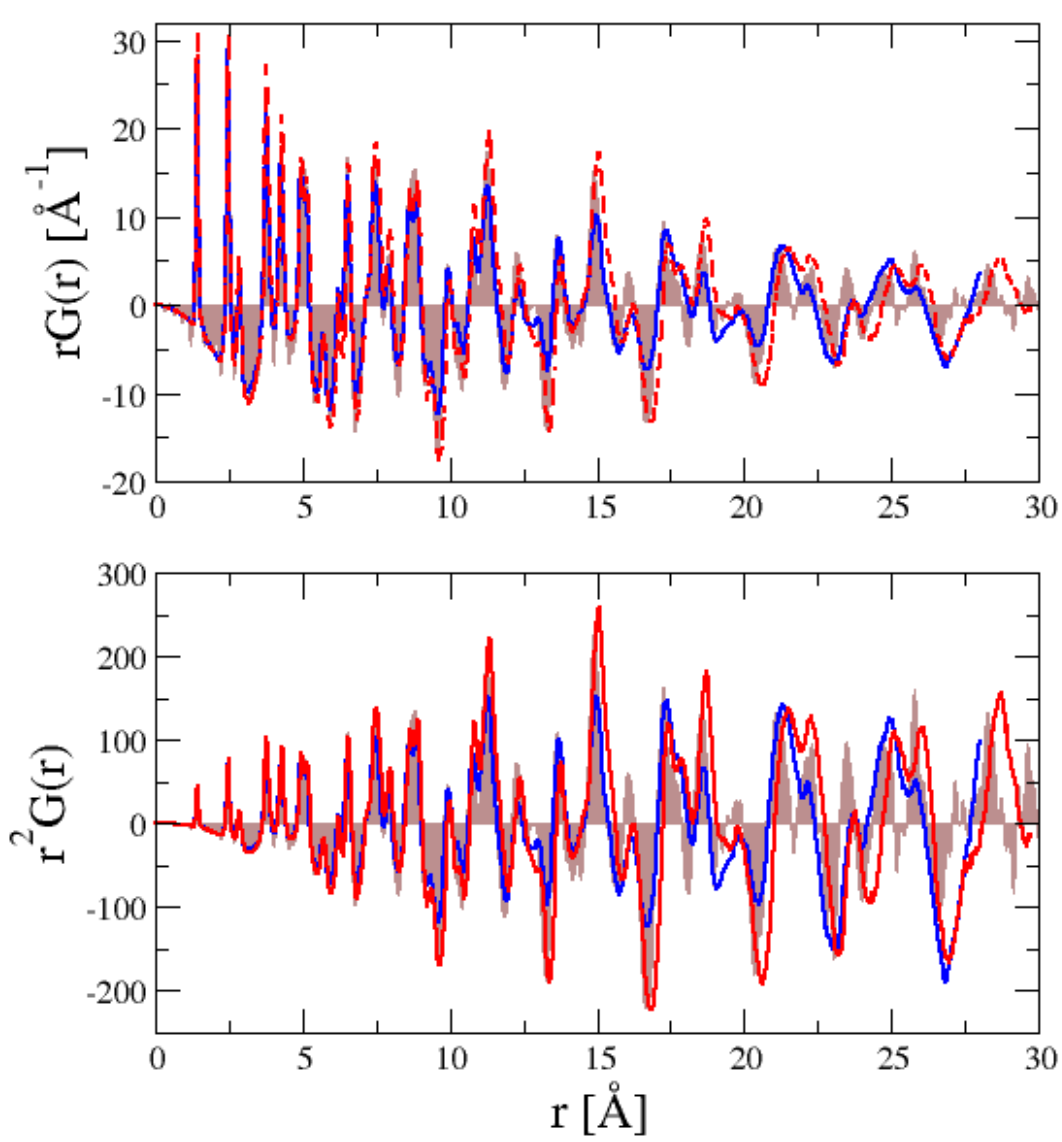

Figure 14: $r G(r)$ (top) and $r^{2} G(r)$ functions of the PyC models and of a real PyC. Same color code as Fig. 14.

Finally, we plot on Fig. 14 the $r G(r)$ (14a) and $r^{2} G(r)$ (14b) functions of the two models and the experimental functions of a RL PyC [10]. These two figures confirm that both models reproduce reasonably well the Neutron diffraction data and that somehow, especially when looking at intermediate interatomic distances (from 5 to $25 \AA$ ), the experimental curves of $G(r)$, 
$r G(r)$ and $r^{2} G(r)$ lie in between the ones of the AP and HT models, confirming the impression from HRTEM observations. Also, looking at Fig. 14b, we see that both models have the "right arrow with constant tail" envelope of $r^{2} G(r)$, which, according to us, is a specificity of rough laminar pyrocarbons [10].

\section{Conclusion}

The image guided atomistic reconstruction (IGAR) method aiming at building realistic models of nanotextured carbons from experimental HRTEM imaging, image processing and atomistic simulation, has been presented in details. After discussing methodological aspects, both in terms of the technique itself and of the tunable parameters inherent to the approach (quench rate, balance between image and interatomic interactions), this method has been applied to build models based on images taken from a rough laminar pyrocarbon matrix of a $\mathrm{C} / \mathrm{C}$ composite, as prepared and heat treated. The structural similarities and differences between the two models have been carefully exposed. In particular, this work confirms a common idea on PyCs, namely that they are essentially made of nanosized aromatic domains packed together in turbostratic arrangements (the BSUs of Oberlin [3]). It also shows that the different sheets of a stack are probably connected to each other (and to those of neighboring stacks) through many screw dislocations in a kind of "car park access ramp" arrangement. This work also shows that the main source of defects in these materials (apart from the screw dislocations) is not $s p^{3}$ carbon atoms as one could first think but non-hexagonal $s p^{2}$ carbon atoms, mainly pentagons and heptagons. In this kind of materials, 
with sheets of relatively low curvatures, pentagons and heptagons (especially pentagons actually) are not found as isolated defects but usually merge as pentagon/heptagon pairs, as well as lines or networks of pentagon/heptagon pairs, defining some kind of intrasheet grain boundaries between disoriented aromatic domains. Although the differences between the structures of the AP and HT models have been here mainly discussed on a qualitative basis, it seems to come out that the HT model shows larger monolayer aromatic domains, larger pentagon/heptagon networks and fewer dislocations.

It is important to recall here that the results presented here are just a first attempt to determine the structure of such complex materials at the atomic scale and that they are probably not definitive results for AP and HT rough laminar PyCs. There is surely a lot of room for improving the IGAR approach and, for instance, work is already in progress to lower the quench rates, and so improve the convergence of the models, by optimizing and parallelizing the code. Also, we are working at including given amounts of hydrogen in the models. Nevertheless, the comparison of the pair distribution functions computed from these models to the data obtained using neutron powder diffraction on a rough laminar $\mathrm{PyC}$ sample clearly validates the interest of image guided approaches. Indeed, the agreement between the modeled and experimental $G(r), r G(r)$ and $r^{2} G(r)$ is particularly good, taking into account that the models have been constructed from only the HRTEM images of the materials and a few, easy to access, experimental data (the density and $d_{002}$ ). In other words, the IGAR approach is able to build a proper structural model of the material from information on its nanotexture only. Other approaches, like the RMC method, usually fail in 
finding a unique nanotexture from structural experimental data (compare for instance the work of Acharya et al. [46] and Smith et al. [43] who have proposed drastically different atomistic models yet reproducing the same PDF data of a nanoporous carbon[47]). We add that, very recently, some atomistic models of soot particles have been proposed through identification of fringes by pattern recognition techniques on HRTEM images, extension to 3D and replacement by polycyclic aromatic molecules [48]. This approach is interesting as it allows to generate models of very disordered materials while at present time the IGAR method requires some particular symmetry in the system to produce 3D image blocks from 2D experimental images. Also the atomistic construction in this method is probably much less costly than the very long liquid quench simulations required in the IGAR method. On the opposite, in the IGAR method, no constraint exists on the chemical nature of the material: atoms just locate themselves in space to form the lowest energy structure for a given nanotexture, thanks to the 3D image and the interatomic potential. Finally, if the first interest of the IGAR method is to produce atomistic models, allowing for a subsequent evaluation of many of their physico-chemical properties (mechanical, thermal, et...), this approach can also allow investigating the relationship between the atomistic structure and the image properties (see for instance our qualitative discussion, at the end of section 3.3.4, on the relationship between the screw dislocations observed in the AP model and the blurred areas observed in the corresponding simulated images). This will be investigated more thoroughly in future work. 


\section{Acknowledgements}

Funding from the Agence Nationale de la Recherche through the PyroMaN project (contract ANR-2010-BLAN-929) and from the Institut Carnot Materials and systems Institute of Bordeaux (MIB) are gratefully acknowledged.

\section{References}

[1] Manocha LM, Fitzer E. Carbon reinforcements and C/C composites 1st ed. Berlin: Springer; 1998.

[2] Naslain R. Design, preparation and properties of non-oxide CMCs for application in engines and nuclear reactors: An overview. Compos Sci Technol. 2004;64(2):155-70.

[3] Oberlin A. Pyrocarbons. Carbon. 2002;40:7-24.

[4] Reznik B, Hüttinger KJ. On the terminology for pyrolytic carbons. Carbon. 2002;40(4):617-36.

[5] Vallerot JM, Bourrat X, Mouchon A, Chollon G. Quantitative structural and textural assessment of laminar pyrocarbons through Raman spectroscopy, electron diffraction and few other techniques. Carbon. 2006;44(9):1833-44.

[6] Dong GL, Hüttinger KJ. Consideration of reaction mechanisms leading to pyrolytic carbon of different textures. Carbon. 2002;40(14):2515-28.

[7] Bourrat X, Langlais F, Chollon G, Vignoles GL. Low temperature pyrocarbons: A review. J Braz Chem Soc. 2006;17(6):1090-5. 
[8] Reznik B, Gerthsen D, Hüttinger KJ. Micro- and nanostructure of the carbon matrix of infiltrated carbon fiber felts. Carbon. 2001;39(2):21529.

[9] Bourrat X, Lavenac J, Langlais F, Naslain R. The role of pentagons in the growth of laminar pyrocarbon. Carbon. 2001;39(15):2369-86.

[10] Weisbecker P, Leyssale JM, Fischer HE, Honkimäki V, Lalanne M, Vignoles GL. Microstructure of pyrocarbons from pair distribution function analysis using neutron diffraction. Carbon. 2012;50(4):1563-73.

[11] Biggs MJ, Buts A. Virtual porous carbons: what they are and what they can be used for. Mol Sim. 2006;32(7):579-93.

[12] Marks NA, McKenzie DR, Pailthorpe BA, Bernasconi M, Parrinello M. Microscopic structure of tetrahedral amorphous carbon. Phys Rev Lett. 1996;76:768-71.

[13] Marks NA, Cooper NC, McKenzie DR, McCulloch DG, Bath P, Russo SP. A comparison of density functional, tight-binding and empirical methods for the simulation of amorphous carbon. Phys Rev B. 2002;65:075411.

[14] Kumar A, Lobo RF, Wagner NJ. Porous amorphous carbon models from periodic Gaussian chains of amorphous polymers. Carbon. 2005;43:3099-111.

[15] Roussel T, Didion A, Pellenq RJM, Gadiou R, Bichara C, Vix-Guterl C. Experimental and atomistic simulation study of the structural and 
adsorption properties of Faujasite zeolite-templated nanostructured carbon materialst. J Phys Chem C. 2007;111(43):15863-76.

[16] McGreevy RL, Pusztai L. Reverse Monte Carlo simulation: A new technique for the determination of disordered structures. Mol Sim. 1988;1:359-67.

[17] Jain SK, Pellenq RJM, Pikunic JP, Gubbins KE. Molecular modeling of porous carbons using the hybrid reverse Monte Carlo method. Langmuir. 2006;22(24):9942-8.

[18] Petersen TC, Yarovsky I, Snook IK, McCulloch DG, Opletal G. Structural analysis of carbonaceous solids using an adapted reverse Monte Carlo algorithm. Carbon. 2003;41(12):2403-11.

[19] Nguyen TX, Cohaut N, Bae JS, Bhatia SK. New method for atomistic modelling of the microstructure of activated carbons using hybrid reverse Monte Carlo simulation. Langmuir. 2008;24(15):7912-22.

[20] Pikunic J, Clinard C, Cohaut N, Gubbins KE, Guet JM, Pellenq RJM, et al. Structural modeling of porous carbons: constrained reverse Monte Carlo method. Langmuir. 2003;19(20):8565-82.

[21] Palmer JC, Llobet A, Yeon SH, Fischer JE, Shi Y, Gogotsi Y, et al. Modeling the structural evolution of carbide-derived carbons using quenched molecular dynamics. Carbon. 2010;48(4):1116-23.

[22] Palotás AB, Rainey LC, Feldermann CJ, Sarofim AF, Vander Sande JB. Soot morphology: An application of image analysis in high-resolution transmission electron microscopy. Micros Res Tech. 1996;33(3):266-78. 
[23] Shim HS, Hurt RH, Yang NYC. A methodology for analysis of 002 lattice fringe images and its application to combustion-derived carbons. Carbon. 2000;38(1):29-45.

[24] Rouzaud JN, Clinard C. Quantitative high-resolution transmission electron microscopy: a promising tool for carbon materials characterization. Fuel Process Technol. 2002;77-78:229-35.

[25] Germain C, Da Costa JP, Lavialle O, Baylou P. Multiscale estimation of vector field anisotropy application to texture characterization. Signal Process. 2003;83(7):1487-503.

[26] Vander Wal RW, Tomasek AJ, Pamphlet MI, Taylor CD, Thompson WK. Analysis of HRTEM images for carbon nanostructure quantification. J Nanopart Res. 2004;6:555-68.

[27] Portilla J, Simoncelli EP. A parametric texture model based on joint statistics of complex wavelet coefficients. Int J Comput Vis. 2000;40(1):49-71.

[28] Da Costa JP, Germain C. Synthesis of solid textures based on a 2D example: application to the synthesis of 3D carbon structures observed by transmission electronic microscopy. In: Proceedings of SPIE, the International Society for Optical Engineering;. .

[29] Leyssale JM, Da Costa JP, Germain C, Weisbecker P, Vignoles GL. An image guided atomistic reconstruction of pyrolytic carbons. App Phys Lett. 2009;95(23):231912. 
[30] Da Costa JP, Germain C, Baylou P, Cataldi M. An image analysis approach for the structural characterization of pyrocarbons. In: Proceedings of the International Conference on Composite Testing and Model Identification (CompTest 2004). Bristol, UK; 2004. .

[31] Brenner DW, Shenderova OA, Harrison JA, Stuart SJ, Ni B, Sinnott SB. A second-generation reactive empirical bond order (REBO) potential energy expression for hydrocarbons. J Phys: Condens Matter. 2002;14(4):783-802.

[32] Petersen TC, Snook IK, Yarovsky I, McCulloch DG, O’Malley B. Curved-surface atomic modeling of nanoporous carbon. J Phys Chem C. $2007 ; 111(2): 802-12$.

[33] Allen MP, Tildesley DJ. Computer Simulation of Liquids. Oxford University Press; 1987.

[34] Stuart SJ, Tutein AB, Harrison JA. A reactive potential for hydrocarbons with intermolecular interactions. J Chem Phys. 2000;112(14):647286.

[35] Franzblau DS. Computation of ring statistics for network models of solids. Phys Rev B. 1991;44(10):4925-30.

[36] Jain SK, Gubbins KE. Ring connectivity: measuring network connectivity in network covalent solids. Langmuir. 2007;23(3):1123-30.

[37] Kilaas R. Interactive software for simulation of high resolution TEM images. In: Geiss RH, editor. Proceedings of the 22nd Annual Conference of the Microbeam Aanalysis Society; 1987. p. 293-300. 
[38] Petersen TC, Yarovsky I, Snook IK, McCulloch DG, Opletal G. Microstructure of an industrial char by diffraction techniques and reverse Monte Carlo modelling. Carbon. 2004;42(12-13):2457-69.

[39] Kuc A, Seifert G. Hexagon-preserving carbon foams: Properties of hypothetical carbon allotropes. Phys Rev B. 2007;74(21):214104.

[40] Grantab R, Shenoy VB, Ruoff RS. Anomalous strength characteristics of tilt grain boundaries in graphene. Science. 2010;330(6006):946-8.

[41] Jeong BW, Ihm J, Lee GD. Stability of dislocation defect with two pentagon-heptagon pairs in graphene. Phys Rev B. 2008;78(16):165403.

[42] Yazyev OV, Louie SG. Topological defects in graphene: Dislocations and grain boundaries. Phys Rev B. 2010;81(19):195420.

[43] Smith MA, Foley HC, Lobo RF. A simple model describes the PDF of a non-graphitizing carbon. Carbon. 2004;42(10):2041-8.

[44] Harris PJF, Liu Z, Suenaga K. Imaging the structure of activated carbon using aberration corrected TEM. J Phys: Conf Series. 2010;241(1):012050.

[45] Leyssale JM, Vignoles GL, Villesuzanne A. Rippled nanocarbons from periodic arrangements of reordered bivacancies in graphene or SWCNTs. Submitted. 2011;

[46] Acharya M, Strano MS, Mathews JP, Billinge SJL, Petkov V, Subramoney S, et al. Simulation of nanoporous carbons: A chemically constrained structure. Phil Mag B. 1999;79(10):1499-518. 
[47] Petkov V, Difrancesco RG, Billinge SJL, Acharya M, Foley HC. Local structure of nanoporous carbons. Phil Mag B. 1999;79(10):1519-30.

[48] Fernandez-Alos V, Watson JK, vander Wal R, Mathews JP. Soot and char molecular representations generated directly from HRTEM lattice fringe images using Fringe3D. Combustion and Flame. 2011;158(9):1807 -13 . 